\title{
Model-based real-time plasma electron density profile estimation and control on ASDEX Upgrade and TCV
}

\begin{abstract}
Real-time plasma electron density profile estimation and control are essential in the operation of future tokamaks. In particular, the robustness against diagnostics failure and disturbances is important for long pulse operation.

A model-based approach to profile estimation is implemented on the control systems of ASDEX Upgrade and TCV, which is able to merge information from various diagnostics for both core and edge density, as well as systematically handling diagnostic failure. The model used for profile estimation is employed to tune a feedback controller before an experiment, thereby reducing the experimental time required for tuning.

Subsequently, this observer and controller have been employed in scientific experiments on ASDEX Upgrade and TCV. On ASDEX Upgrade, the density profile estimator was used in high-density pellet-fuelled discharges, providing a more reliable real-time estimate of the core density for feedback control than previously achieved. On TCV, in experiments on integrated pressure and safety factor profile control, the density profile estimator and feedback controller provide a constant density despite disturbances from time-varying ECCD power. Additionally, the real-time density profiles provide an essential input for other
\end{abstract}

This manuscript is submitted to Fusion Engineering and Design. The authors of the manuscript are T.C. Blanken, F. Felici, C. Galperti, O. Kudláček, F. Janky, A. Mlynek, L. Giannone, P.T. Lang, W. Treutterer, W.P.M.H. Heemels, M.R. de Baar, the TCV team, the ASDEX Upgrade team and the EUROfusion MST1 team. 
real-time plasma state estimation codes including Electron Cyclotron ray tracing codes, contributing to a complete real-time estimation of the entire plasma state.

\section{Introduction}

In future tokamak reactors such as ITER and DEMO, the plasma particle density must be controlled since it directly affects the fusion power, radiation and the non-inductive current distribution [Pironti and Walker, 2005; Wesson, 2004]. Moreover, the density restricts Electron Cyclotron (EC) heating systems (through e.g. EC cutoff) and neutral beam operation (through e.g. beam shinethrough). Additionally, future reactors will necessarily operate close to operational limits (formed by e.g. the Greenwald density limit and tearing modes) which can lead to disruptions. Therefore, these operational limits must be actively avoided, and the requirements for the control of the density on reactors will be increasingly stringent than those on present-day devices [Biel et al., 2015; Gribov et al., 2007].

In particular, the plasma density must be controlled in feedback, since shotto-shot differences and disturbances (e.g. confinement deterioration from (unexpected) MHD activity or varying wall conditions) could otherwise result in deviations from the target density. Feedback control requires an accurate estimate of the controlled density. However, the diagnostics can have failures (e.g. , fringe jumps on interferometers) and have a finite reliability; in particular in a nuclear environment [Biel et al., 2015]. Therefore, the real-time density estimate must be resilient to common failure modes of diagnostics which could otherwise lead to loss of control and early termination of a discharge.

In recent years, successful research has been done to address the various challenges. Research on real-time density estimation has focused on static fitting methods [Fischer et al., 2010; Mlynek et al., 2011]. However, these methods are susceptible to diagnostic faults. Integrated diagnostic analysis methods for full profile estimation using Bayesian inference employing multiple diagnostics (e.g. , interferometry, Thomson scattering, ECE and/or impact excitation spectroscopy) are powerful [Fischer et al., 2010; Galante et al., 2015; Rathgeber et al., 2010; Reusch et al., 2018], but have not been done in real time for control applications. Detection of faults on density diagnostics has focussed on fringe jump detection from interferometers [Gil et al., 2010; Mlynek et al., 2011].

In work on density feedback control, the controlled density signal is often derived from a single diagnostic system or channel, and it is assumed that this measurement is reliable, see e.g. [Janky et al., 2015; Vijvers et al., 2012]. In [Lang et al., 2012], a hardwired switch from the estimate based on DCN laser interferometers [Mlynek et al., 2011] to an estimate based on $\mathrm{CO}_{2} / \mathrm{HeNe}$ laser interferometer was used for density control where pellet injection proved problematic for the DCN system. This paper will provide a generalization of these approaches, 
to allow an arbitrary number of diagnostics to be used and allow handling of a wide range of fault scenarios. In our previous work [Blanken et al., 2018b] (this thesis, Chapter 2), a model-based estimator was introduced for profile estimation, which included fringe jump correction. In this approach, a Kalman filter is used to merge interferometry measurements with a model for the density profile evolution. Thereby, it provides redundancy against diagnostic failure, and promotes smoothness of profile estimates to the extent that is in accordance with the physics-based model. The Kalman filter is a recursive Bayesian filter for linear systems [Anderson and Moore, 1979]. It is also used in the RAPTOR-observer for estimation of the temperature and q profile [Felici et al., 2014a, 2016]. Also, the method allows for fringe jump correction based on mismatches between diagnostic signals and model predictions. This real-time capable model was derived from radial transport and heuristic particle flow (e.g. recycling and molecular processes) modeling, since both full first-principle modeling and modeling based on identification from data is considered too challenging, see [Blanken et al., 2018b]. However, this model-based estimator was not yet employed in experiments. This paper provides the first results of actual application of this method in both TCV and ASDEX Upgrade.

In the existing practice of plasma density feedback control, the controller gains are often manually tuned using trial and error on multiple discharges. This is not practical for ITER and DEMO, where experimental time is costly and limited. Instead, a tuning procedure based on a model of the dynamic response of the density to actuation commands should be used. This allows to tune the controller gains before a discharge, so that the experimental time required for tuning can be reduced significantly.

This paper reports on the implementation of the observer on the real-time control systems of ASDEX Upgrade and TCV for experimental use. Moreover, the model [Blanken et al., 2018b] is employed to derive a feedback controller for TCV using robust $H_{\infty}$ synthesis.

The density profile observer has been employed on ASDEX Upgrade in high-density pellet-fuelled discharges [Lang et al., 2018], which raise the central plasma density above the Greenwald limit. In order to provide diagnostic redundancy, the model-based density profile estimator [Blanken et al., 2018b] is extended with a forward model for the $\mathrm{CO}_{2}$ interferometer and the Bremsstrahlung measurement. While [Lang et al., 2018] presented the scenario development and results, this paper provides more details on the estimator and its diagnostics integration and fault handling.

On TCV, the profile observer is combined with the density feedback controller for use in experiments on profile control [Maljaars et al., 2017]. In these experiments, the controller ensures a constant density in time despite disturbances from feedback-controlled pressure and safety factor profile using ECCD. The profile observer is included in the architecture for integrated plasma con-trol on TCV [Blanken et al., 2018a; Vu et al., 2018] to 
provide the Greenwald density fraction.

The remainder of this paper is structured as follows. In Section 2, a sum-mary of the control-oriented model, the forward diagnostics models and the density profile observer is given. In Section 3, the control problem in the high-density pellet-fuelled discharges, implementation of the observer on the ASDEX Upgrade control system and the density estimation and control are presented. In Section 4, the control problem in profile control experiments, the feedback con-troller design, the implementation of the observer and the controller on the TCV control system, and the results of density control are presented. In Section 5, the methods and experimental results are discussed. Finally, in Section 6, conclusions are presented.

\section{Model-based density profile observer}

A model-based observer for the density profile was introduced in [Blanken et al., 2018b]. It consists of an Extended Kalman filter (EKF) [Anderson and Moore, 1979] which incorporates the RAPDENS model. Moreover, a number of fault handling strategies are employed. A summary of the observer is given in this section.

\subsection{RAPDENS: a control-oriented 0D+1D model of the electron density evolution}

The RAPDENS model [Blanken et al., 2018b] is a control-oriented predictive model for the evolution of the electron density profile $n_{e}(\rho, t)$, vacuum neutral particle inventory and wall inventory. It is a physics-based dynamic selfconsistent model, which includes the effects of:

- particle conservation law for radial (1D) plasma electron evolution,

- OD models for wall and vacuum neutral particle inventories,

- semi-empirical radial diffusion-drift model,

- semi-empirical source models for inter-inventory channels, e.g. recycling and ionization,

- switched model coefficients for limited versus diverted plasmas. Similar for Ohmic or L-mode versus H-mode plasmas,

- parametrized equilibrium-related quantities given as external (time-varying) input,

- low execution time due to specific choices of temporal and spatial discretization. For example, the execution time is about $10 \%$ of the simulation time on the TCV digital control system hardware. 
In this model, the electron density profile inside the confined region of the plasma is parametrized as

$$
n_{e}(\rho, t)=\sum_{i=1}^{m} \Lambda_{i}(\rho) x_{i}(t)
$$

where $\rho$ is the normalized toroidal flux, $\Lambda_{i}(\rho)$ are cubic spline basis functions (see [Blanken et al., 2018b]) and $x_{i}(t)$ is the vector containing the state variables parametrizing the density. A summary of the model equations is given in Appendix A. The full details and derivations are provided in [Blanken et al., 2018b]. For the applications reported in this paper, the dynamics are discretized in space and time similar to equation (3.19) as

$$
f\left(x\left(t_{k}\right), x\left(t_{k-1}\right), p\left(t_{k-1}\right), u\left(t_{k-1}\right)\right)=0
$$

where $x\left(t_{k}\right)$ is the system state at time $t_{k}, p\left(t_{k}\right)$ contains external physics quantities that influence the density evolution such as plasma current, volume and temperature, and $u\left(t_{k}\right)$ is the actuation input such as gas valve flux, pellet particle flux and neutral beam injection.

\subsection{Forward synthetic diagnostic models}

For the application of a state observer, synthetic models for the relations between the system state $x$ and the measured quantities are needed. These forward synthetic diagnostic models are written in a generic form as

$$
y=h(x, \zeta)
$$

where $y$ is the vector of measured quantities and $\zeta$ is a vector containing physical quantities other than the plasma density which may affect the diagnostic measurement. Device-specific functions $h(x, \zeta)$ for the ASDEX Upgrade and TCV diagnostic systems are derived in Section 3.2 and Section 4.3.

\subsection{Profile observer design}

The operation of the observer is explained next, while the relevant equations are provided in full detail in Appendix B. A dynamic state observer for the density profile [Blanken et al., 2018b] incorporates an Extended Kalman filter [Anderson and Moore, 1979] based on the RAPDENS model (Section 2.1) and forward diagnostics models. The Kalman filter is a minimum-variance iterative estimator for linear dynamical systems [Anderson and Moore, 1979]. Since the dynamics in (3.2) are nonlinear, local linearizations are substituted in the Kalman filter equations, resulting in the Extended Kalman filter.

At every time step $t_{k}$ at which the measurement $y_{k}$ becomes available, 
1. a one time step ahead prediction of the state, denoted ${ }^{1}$ as $\hat{x}_{k \mid k-1}$, is computed based on the previous estimate $\hat{x}_{k-1 \mid k-1}$ using the model (3.2),

2. a prediction of the measurement, denoted as $\hat{y}_{k \mid k-1}=h\left(\hat{x}_{k \mid k-1}, \hat{\zeta}_{k-1 \mid k-1}\right)$, is computed based on the predicted state $\hat{x}_{k \mid k-1}$ and $\hat{\zeta}_{k-1 \mid k-1}$ using the forward diagnostic model (3.3),

3. the difference between the measurement $y_{k}$ and the predicted measurement $\hat{y}_{k \mid k-1}$, called the residual $z_{k}$, is computed,

4. the residual is corrected for any detected faults in the diagnostic measurements (discussed in the next subsection),

5. the posterior state estimate $\hat{x}_{k \mid k}$ and $\hat{\zeta}_{k \mid k}$ is computed by summing the predicted state $\hat{x}_{k \mid k-1}$ and $\hat{\zeta}_{k-1 \mid k-1}$ with the corrected residual multiplied by the Kalman gain matrix (see (3.31) and Appendix B),

6. various output signals of the observer are computed, e.g. the estimated density profile by substituting $\hat{x}_{k \mid k}$ in (3.1).

These steps are repeated at each time step. By proper tuning of the observer gain matrix, the state estimate $\hat{x}_{k \mid k}$ converges to the actual (not directly measured) state $x_{k}$ of the system. In Figure 3.1, a block diagram of the observer is shown. This diagram illustrates the steps above, as well as its input and output signals, while the underlying equations are provided in Appendix B.

\section{$2.4 \quad$ Fault handling}

Since information of the observer is used by the density controller and other real-time algorithms, errors in the observer results due to diagnostic faults may propagate downstream to the controller and other real-time algorithms. This can have negative consequences for the discharge. Therefore, the density profile estimates should not become invalid following diagnostics faults and faults on other input signals. Typical examples of diagnostic faults are fringe jumps on interferometers due to fast density transients and signal degradation due to hardware malfunction. Moreover, other real-time algorithms can experience numerical issues and might provide invalid inputs for the observer.

In the observer implementations on ASDEX Upgrade and TCV, two types of fault handling are considered:

1. diagnostics fault handling by real-time detection and correction of known fault modes, and

2. fault handling of the parameter values $p\left(t_{k}\right)$ by only passing values that are within specified credibility limits.

First, information about diagnostic fault detection in the ASDEX Upgrade digital control system (DCS) is communicated via dedicated validity flags for each

\footnotetext{
${ }^{1}$ The variable $\hat{x}_{i \mid j}$ denotes the estimate of quantity $x$ at time $i$, given measurements available up to time $j$.
} 


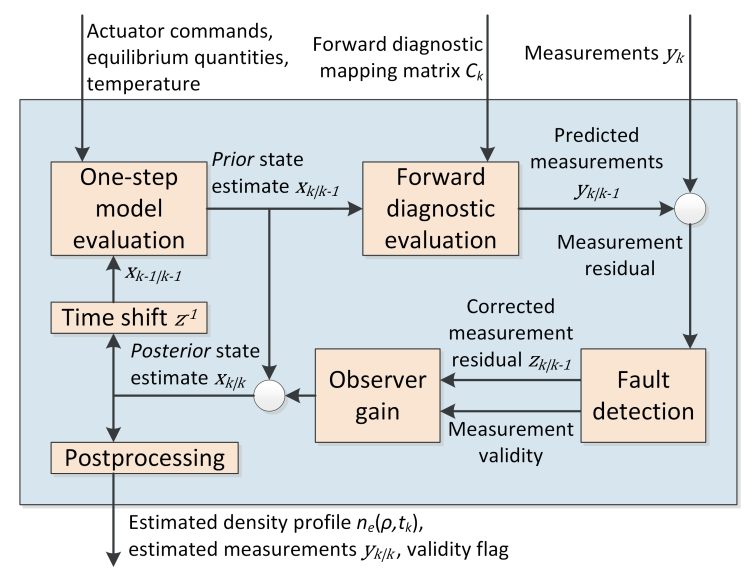

Figure 3.1 - Block diagram of the RAPDENS profile observer. This diagram illustrates the recursive Extended Kalman filter equations provided in Appendix B as well as the fault detection from Section 2.4. The observer receives measurements, fuelling commands, as well as the forward diagnostic mapping matrix, plasma current, volume and temperature. At every iteration of the observer, a one-sample ahead prediction is made based on the nonlinear model (3.2) and a forward diagnostics model (3.3) given the state estimate at the previous time step. The state estimate is updated with information from the measurement sample $y_{k}$.

real-time signal (see [Treutterer et al., 2014a]). If a diagnostic signal has a validity flag that is not 'good' or the signal is not being produced, the corresponding entries are ignored in the observer equations. Moreover, both implementations have detection and correction schemes for fringe jumps on interferometers. This is shown in more detail in Section 3.2, 4.3 and Appendix B.

Second, faults on input signals are detected by comparing the signal value to pre-configured validity limits. If a signal value is considered invalid, the previous valid value is held or a user-defined backup value is substituted. For example, if the signal of the real-time reconstructed plasma volume is below or above credible limits (e.g. due to invalid information from the real-time equilibrium reconstruction), the previous credible value is held. Examples of the handling of fault events in experiments are shown in Section 3.3.

\section{Profile estimation in high-density experiments on ASDEX Upgrade}

In this section, the implementation of the observer on ASDEX Upgrade and its estimation performance in experiments are presented. 


\subsection{Plasma scenario and control problem}

In high-density scenarios on ASDEX Upgrade, a core density above the Greenwald limit is reached through pellet injection [Lang et al., 2018]. The scenario is a diverted H-mode at a central density of about $1.6 \times 10^{20} \underset{\mathrm{m}^{\underline{3}}}{\#^{3}}$ corresponding to a Greenwald fraction of $f_{\mathrm{GW}}=1.25$.

The central electron density is required to follow a step reference signal during the current flat-top phase. Reference tracking is achieved by a feedback controller that commands the pellet launcher as the actuator, see [Lang et al., 2018]. The controlled quantity is provided in real time by the density observer, which can be either the core density, volume- or profile-averaged density. This observer should:

- minimize the profile estimation error, in particular the estimation error of the central density,

- detect and correct for measurement faults,

- be robust against model mismatches.

Moreover, the observer implementation should stay within the available computational time per cycle time of the real-time computer. The cycle time of the DCS is either $1 \mathrm{~ms}$ or $1.5 \mathrm{~ms}$.

\subsection{Implementation on the ASDEX Upgrade control sys- tem}

The observer from Section 3.2 is programmed in MATLAB Simulink [The Mathworks Inc., a], then automatically converted into $\mathrm{C}$ code for implementation in the DCS [Treutterer et al., 2014a]. A block scheme of the implementation of the density profile observer on the DCS is shown in Figure 3.2. The observer receives:

- interferometer and Bremsstrahlung measurements (discussed next),

- a forward interferometer model $C_{\mathrm{FIR}}$ (discussed next) from the real-time equilibrium reconstruction JANET [Giannone et al., 2015],

- equilibrium information (plasma current $I_{\mathrm{p}}$, plasma volume $V_{\mathrm{p}}$, normalized poloidal flux $\rho_{\text {pol }}\left(\rho_{\text {tor }}\right)$ and x-point presence indicator $\left.c_{\mathrm{LD}}\right)$ from JANET [Giannone et al., 2015],

- the estimated plasma electron temperature $T_{\mathrm{e}}$ from the RAPTOR observer [Felici et al., 2011, 2014a].

The quantities in the latter two points are contained in the parameter $p$, see Section 2.1. In the experiments reported in this paper, the algorithm is run at a cycle time of $1.5 \mathrm{~ms}$. 


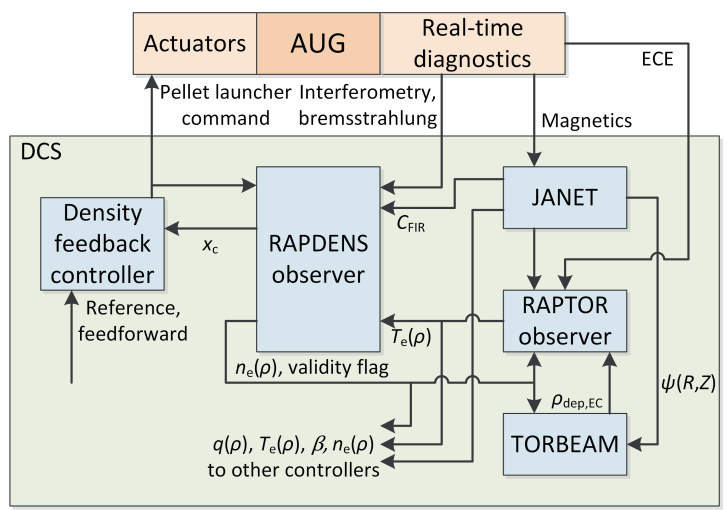

Figure 3.2 - Block diagram of the interconnection of the density profile observer on the ASDEX Upgrade digital control system (DCS) [Treutterer et al., 2014a]. The observer (see Figure 3.1) receives interferometry and bremsstrahlung measurements, a temperature profile from the RAPTOR-observer as well as the interferometry matrix $C_{\mathrm{FIR}}$, plasma current and volume from the equilibrium solver. In the experiment discussed in Section 3.3, the estimated on-axis density is the controlled variable $x_{\mathrm{c}}$ which is provided to the feedback controller by the observer.

\section{Forward diagnostics modeling}

The diagnostics available in real time on ASDEX Upgrade are the six interferometer chords and two Bremsstrahlung measurement channels. Forward diagnostic models are constructed, which relate the state of the system to the measured quantities as expressed in Section 2.2.

The interferometers measure a line-integrated density. This measurement may be corrupted by fringe jumps, and can be written as $y_{\mathrm{DCN}}=\int_{L_{\mathrm{DCN}}} n_{e} d L+$ $\delta d$, where $\delta$ is a constant equal to the line-integrated density corresponding to one fringe and $d$ is an integer representing the cumulative number of fringe jumps at any point in time.

On the DCS, a similar calculation is done by a postprocessing routine of the real-time equilibrium reconstruction code [Giannone et al., 2015], but for the monomial basis functions defined on $\rho_{\text {pol }}$ used in [Mlynek et al., 2011]. Using time-varying equilibria, this result is mapped to the basis functions on $\rho_{\text {tor }}$ considered in this work (i.e. (3.1)). This results in a linear algebraic relation between the state $x$ and the measurement $y_{\mathrm{DCN}}$.

Since the line-integrals for the V1 interferometer line-of-sight are currently not evaluated in real-time, a different approach is taken to construct this forward model. The measured signal of V1 is assumed to be proportional to the volume-averaged density and modeled as $y_{\mathrm{DCR}} \mathrm{V} 1=\zeta_{\mathrm{V} 1} \stackrel{1}{\mathrm{p}}^{V_{\mathrm{p}}} n_{e} d V$ where $\zeta_{\mathrm{V} 1}$ 
is a slowly-changing coefficient that is to be estimated by the observer. Also, the electronics of $\mathrm{V} 1$ is naturally resilient to fringe jumps because of its long wavelength [Mlynek et al., 2012].

The Bremsstrahlung depends on effective charge, electron temperature and density as $S_{\mathrm{B}} \propto Z_{\mathrm{eff}} T_{e}^{1 / 2} n_{e}^{2}$ [Wesson, 2004]. On ASDEX Upgrade, the Bremsstrahlung is measured along two lines of sight tangential to the toroidal direction. A realtime evaluation of the density basis functions over the tangential line of sight was not available, therefore a different approach was taken. Instead, it is assumed that the density dependence is a volume-averaged density dependence. Moreover, it is assumed that the effective charge and temperature change slowly with respect to the density. Accordingly, the Bremsstrahlung measurement equation is written as $y_{\mathrm{Br}}=\zeta_{\mathrm{Br}}\left(\frac{1}{V_{\mathrm{p}}} \int_{V_{\mathrm{p}}} n_{e} d V\right)^{2}$ where $\zeta_{\mathrm{Br}}$ is a slowly varying coefficient that is to be estimated by the observer. Substituting the density parametrization (3.1) yields the algebraic relation between the state $x$ and the measurement $y_{\mathrm{Br}}$.

The above forward diagnostics models are stacked as

$$
y=\left[\begin{array}{c}
y_{\mathrm{DCN} \_\mathrm{H} 1} \\
\vdots \\
y_{\mathrm{DCN} \_\mathrm{H} 5} \\
y_{\mathrm{DCR} \_\mathrm{V} 1} \\
y_{\mathrm{Br} 1} \\
y_{\mathrm{Br} 2}
\end{array}\right]=\left[\begin{array}{c}
C_{\mathrm{FIR}} x+\delta d \\
\zeta_{\mathrm{V} 1} C_{\mathrm{p}} x \\
\zeta_{\mathrm{Br} 1} x^{\mathrm{T}} C_{\mathrm{p}}^{\mathrm{T}} C_{\mathrm{p}} x \\
\zeta_{\mathrm{Br} 2} x^{\mathrm{T}} C_{\mathrm{p}}^{\mathrm{T}} C_{\mathrm{p}} x
\end{array}\right]
$$

where $C_{\mathrm{FIR}}^{(i, j)}=\int_{L_{\mathrm{DCN} \_\mathrm{H} i}} \Lambda_{j} d L$ and $C_{\mathrm{p}}^{(i)}=\frac{1}{V_{\mathrm{p}}} \int_{V_{\mathrm{p}}} \Lambda_{i} d V$.

\section{Fault handling}

In the real-time implementation, all input signals to the RAPDENS-observer are checked for validity. If a diagnostic sign is labeled valid ${ }^{2}$, the observer uses it as shown in Section 2.3. However, whenever a diagnostic signal is invalid, that diagnostic signal is ignored by the observer at that step in time. This is done by excluding the signal and its entry in the forward diagnostic model (3.4) from the observer equations (see Appendix B) at that time step. In a similar way, the V1 interferometer signal is ignored whenever the total ICRH power exceeds $100 \mathrm{~kW}$, since the ICRH system produces electromagnetic interference on the V1 electronics, rendering the V1 signal invalid.

When large pellets are injected, the H1-H5 interferometers are often rendered unusable: numerous fringe jumps occur due to pellet-induced density transients.

\footnotetext{
${ }^{2}$ In this context, a signal is considered valid if it has the Confidence state good or corrected and the Production state running. Likewise, it is considered invalid if it has Confidence state corrupt, raw or invalid, or has Production state outdated, timeout or stopped. See [Treutterer et al., 2014a].
} 
An example of this is shown in Section 3.3. The H1-H5 interferometers are considered invalid when pellets injection starts.

Moreover, the validity flags of the forward interferometry matrix $C_{\mathrm{FIR}}$ and the signals in $p$, are checked. Whenever a signal is labeled invalid, the previous (valid) signal value is held.

Moreover, a detection scheme for fringe jumps is employed. The original fringe jump detection scheme developed in [Blanken et al., 2018b] did not prove successful for a set of ASDEX Upgrade discharges. This was due to two reasons: density transients (e.g. ramping density) resulted in false positive detection of fringe jumps, and fringe jumps do not present themselves as sharp step-like characteristic on the DCN signals available on the control system but represent as a transient step signal that takes a small number of samples (2 to 4 samples). Therefore a novel detection scheme was developed, which is described next.

In order to detect a fringe jump, each channel of the DCN interferometer measurements is fed through a finite impulse response filter. This filter outputs the difference between two lines fitted on two consecutive time windows. Therefore, this filter lets steps in the signal pass, but filters out ramps and filters out the moving average: slow transients of the density are filtered out, whereas the filter output is sensitive to fringe jumps.

The filter design is best understood when formulated as a least-squares minimization problem. At every cycle of the observer, two lines of equal slope are fitted on a finite history of the measurement $y_{k-2 m-1}, y_{k-2 m}, \ldots, y_{k}$. The line $l_{1}(k)=a_{1}+c k$ is fitted on the measurement series $y_{k-m}, y_{k-m+1}, \ldots, y_{k}$, and the line $l_{2}(k)=a_{2}+c k$ is fitted on the measurement series $y_{k-2 m-1}, y_{k-2 m}, \ldots, y_{k-m-1}$. Note that both series have length $m$ and the lines have equal slope $c$. If the vertical distance between the lines exceeds one fringe, it is assumed that a fringe jump has occurred at time point $k-m$. This vertical distance equals $a_{2}-a_{1}$ and is derived from the solution of the least-squares problem

$$
J_{k}=\min _{a_{1}, a_{2}, c}\left(\sum_{i=k-m}^{k}\left(y_{i}-l_{1}(i)\right)^{2}+\sum_{i=k-2 m-1}^{k-m-1}\left(y_{i}-l_{2}(i)\right)^{2}\right)
$$

The vertical gap at time $k$ is denoted as $a_{k}=a_{2, k}-a_{1, k}$ and is derived from the solution of (3.5). It is a linear combination of the measurement series $y_{k-2 m-1}, y_{k-2 m}, \ldots, y_{k}$. Therefore this is implemented as a finite-impulse response filter.

Finally, the estimated number of fringes that is jumped at time step $k$ is the quotient of the division with remainder of $a_{k}$ by $\delta$ and is expressed as

$$
\hat{\Delta}_{k}=\frac{a_{k}-\left(a_{k} \bmod \delta\right)}{\delta}
$$

where $\bmod (\cdot)$ denotes the modulo operation, i.e. $x \bmod y$ is the remainder after division of $x$ by $y$. 
In the implementation on the DCS, a window length of $m=10$ is chosen. This results in a detection delay of $m=10$ samples, but makes the scheme robust to signal noise.

\subsection{Experimental results of profile estimation}

\section{Profile estimation with fringe jump correction}

In this section, the real-time estimation results on a ASDEX Upgrade discharge with fringe jumps is presented. In discharge \#34188, two fringe jumps occur on edge interferometer channels. These are detected and corrected for, while a number of false detections occur due to fast signal transients resembling fringe jumps.

In Figure 3.3, time traces of the diagnostic signals are shown, together with the estimated measured quantities (see (3.34) of Appendix B). The compari-son of the estimated profiles with offline available Thomson scattering data in Figure 3.4 indicates good estimation accuracy, despite the false jump detections. The pedestal density gradient is modeled by an appropriate reduction of the diffusivity in the model, see Appendix A and [Blanken et al., 2018b].

These correct and false detections are shown in more detail in Figure 3.5. The observer is able to detect and correct for the two fringe jumps, while two false detections take place in a transient phase of the discharge. This is shown in more detail in Figure 3.5. Whereas the first two jumps are detected by a clear shift of the signal, the two false detections are caused by a sudden decrease preceded by a less sudden increase. The fast signal decrease is flagged as a fringe jump.

\section{Profile estimation and control with fault handling}

In this subsection, the real-time estimation results for a high-density scenario with NBH and ICRH is presented. This scenario (see [Lang et al., 2018]) was developed to reach a central density above the Greenwald limit using feedback control on pellet fuelling. Here, the results are presented for a different discharge than in [Lang et al., 2018].

In Figure 3.6, time traces of the diagnostics as well as real-time forward estimated measured quantities are shown for ASDEX Upgrade discharge \#34114. Electromagnetic interference between the ICRH launcher and the V1 interferometer causes the latter to produce invalid signals when the ICRH is on. During the pellet injection phase, all interferometers feature fringe jumps.

Depending on the scenario on ASDEX Upgrade, pellet injection causes one or more interferometers to produce fringe jumps. On the interferometer signals sampled at the DCS cycle time of $1.5 \mathrm{~ms}$, this fast increase resembles a fringe jump. However, not all launched pellets arrive in the plasma. Some pellets may be partially disintegrated before reaching the plasma. Also, in some cases the 

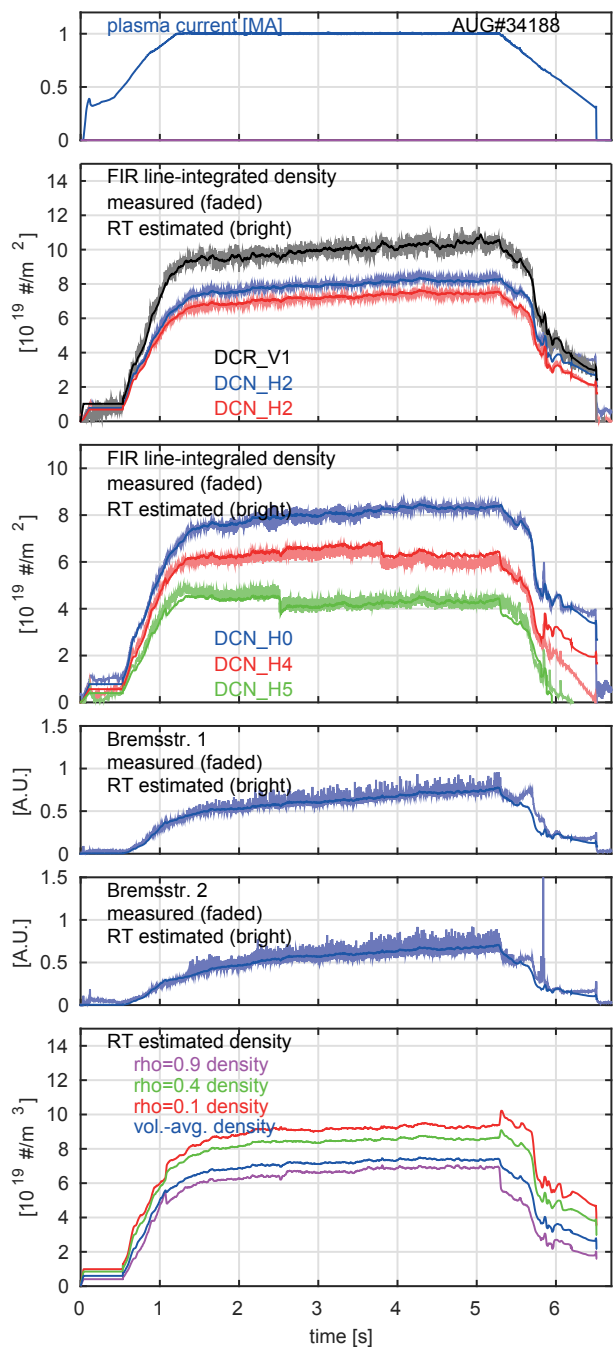

Figure 3.3 - Real-time results for AUG shot \#34188. First panel: plasma current. Second to fifth panel: measured signals of the interferometers and Bremsstrahlung together with the real-time estimated diagnostics signals (computed from the estimated density profile). Sixth panel: real-time estimated density traces. Two fringe jumps occur around $t=2.5 \mathrm{~s}$ and $t=3.8 \mathrm{~s}$ on interferometers H5 and H4, respectively. These are detected and corrected for, as the real-time estimated forward interferometer signals follow these jumps while the estimated density is not affected. The falsely detected jumps around 5.2s and $5.9 \mathrm{~s}$. These affect the estimated density traces, but do not lead to significantly incorrect profiles as can be seen in Figure 3.4. 

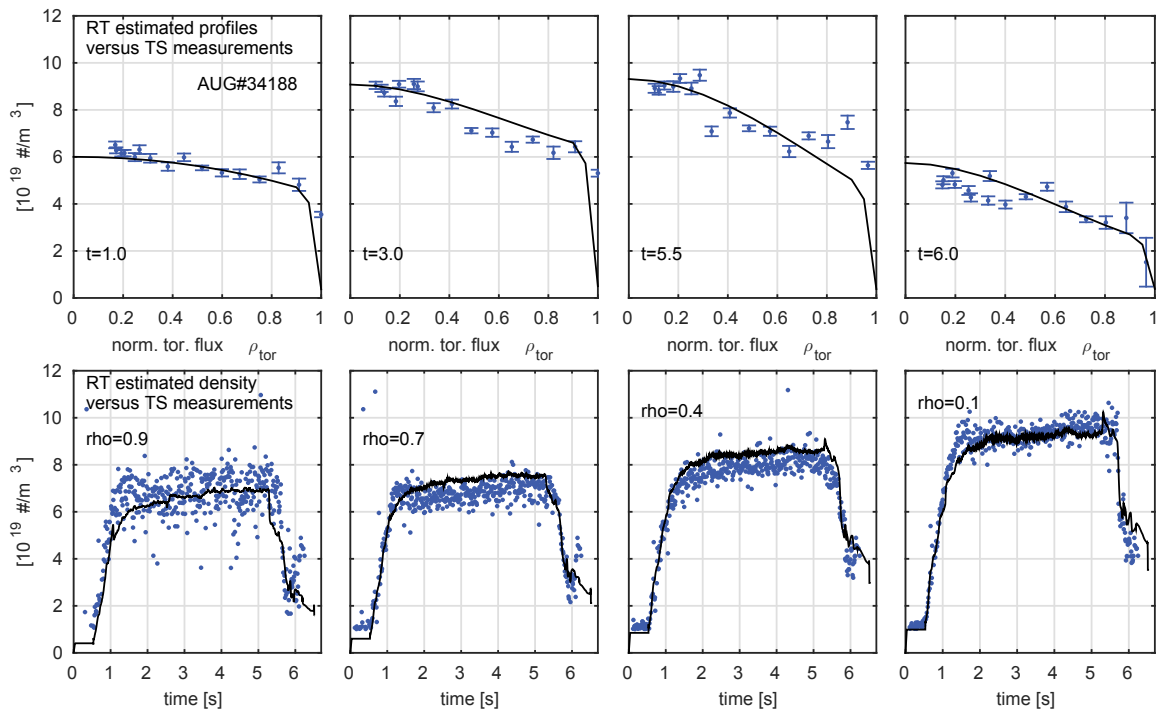

Figure 3.4 - Real-time estimated density profiles with Thomson Scattering measurements for ASDEX Upgrade shot \#34188. Top row: estimated profiles at four points in time. Bottom row: time traces at four loci of the normalized toroidal flux $\rho_{\text {tor }}$. Despite the falsely detected fringe jumps on the edge interferometers shown in Figure 3.3, the estimated profiles show good agreement with the offline available Thomson scattering measurements.
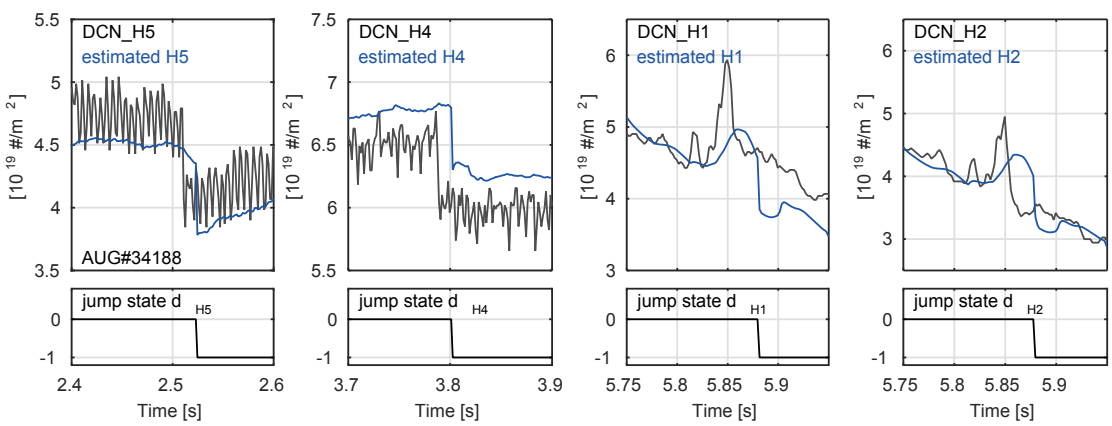

Figure 3.5 - Detailed view of detected and falsely detected fringe jumps in ASDEX Upgrade shot \#34188. Top row: measured interferometer signals $y_{\mathrm{DCN}, k}$ and the real-time synthetic interferometer estimates $\hat{y}_{\mathrm{DCN}, k \mid k}$. Bottom row: estimated jump state $\hat{d}_{k}$. The left two columns are examples of successful fringe jump detection, while the right columns are false detections. The false detections are caused by steep transients in the interferometer signals. Note that an unavoidable delay between signal jump and detection is present. 

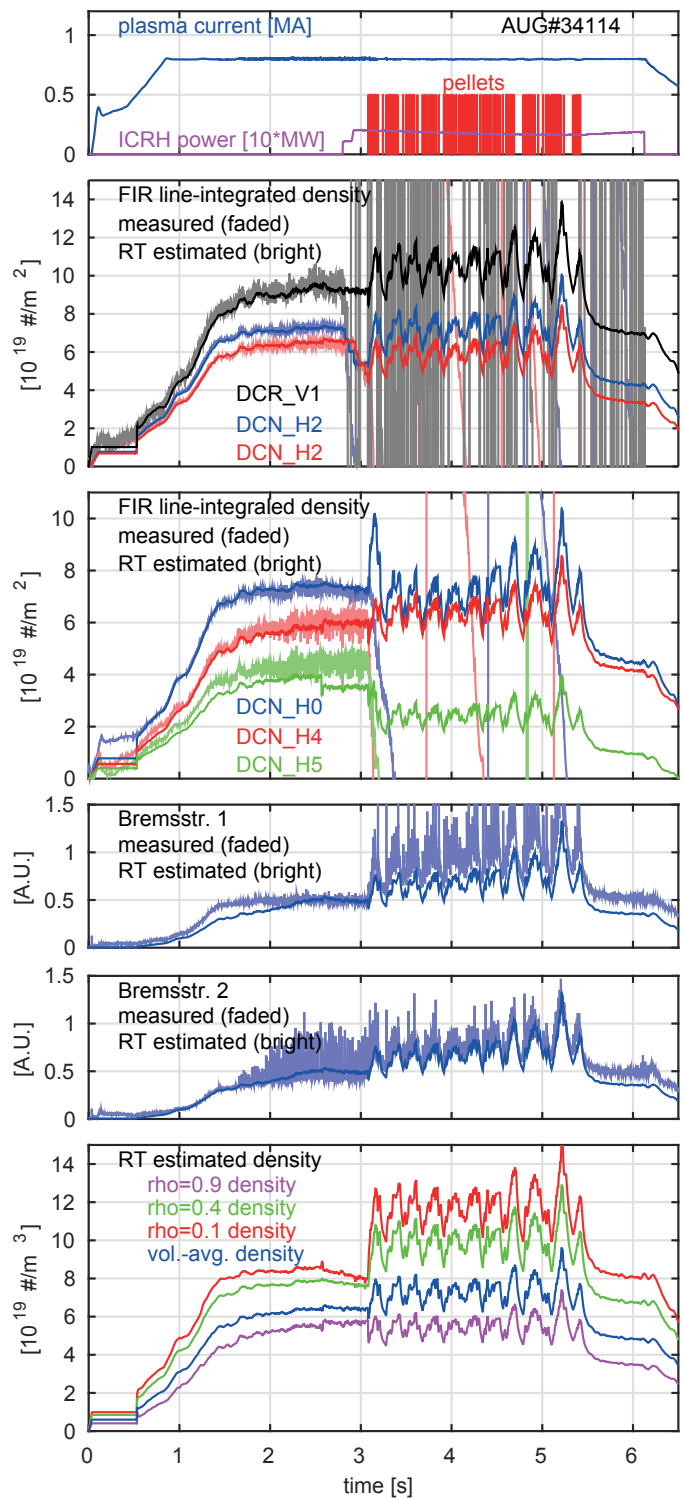

Figure 3.6 - Real-time results for ASDEX Upgrade shot \#34114. Top panel: plasma current, total ICRH power and pellet firing triggers. Second to fifth panel: measured signals of the interferometers and Bremsstrahlung together with the real-time estimated diagnostics signals. Sixth panel: real-time estimated density traces. 

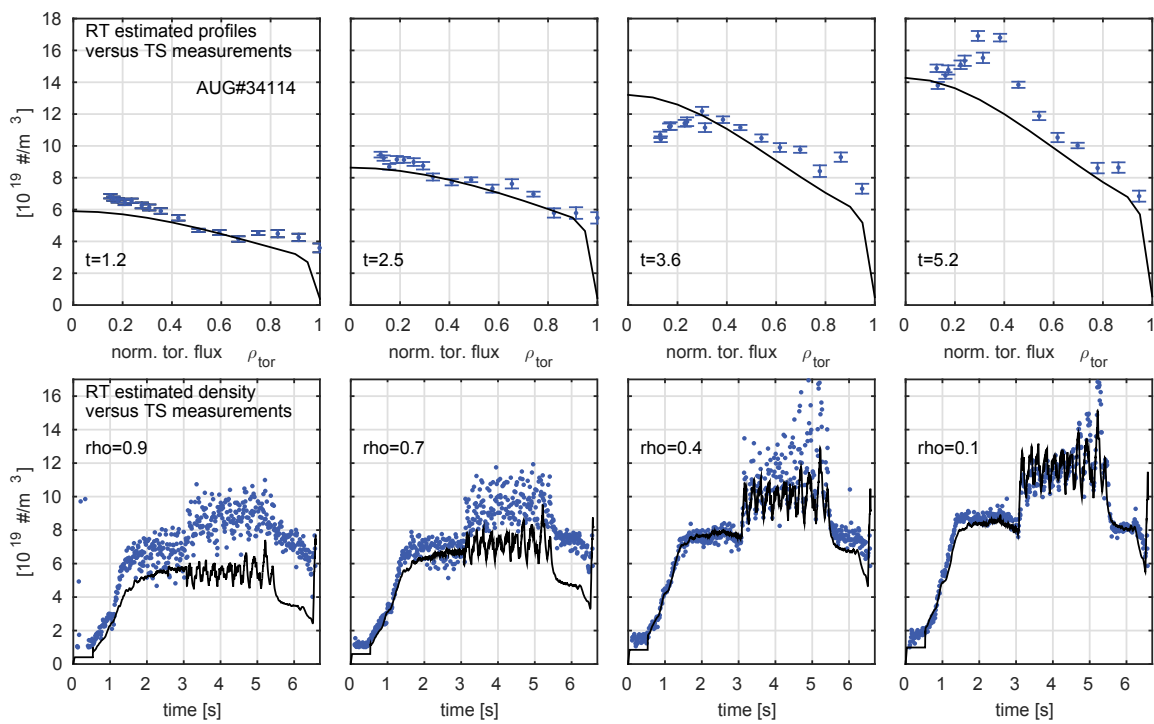

Figure 3.7 - Comparison of the real-time estimated density profiles with offline Thomson Scattering measurements for ASDEX Upgrade discharge \#34114. Top row: estimated profiles at four points in time. Bottom row: time traces at four loci of the normalized toroidal flux $\rho_{\text {tor }}$. The estimation accuracy at the plasma core is good. However, the pedestal density is systematically underestimated. Also note that the observer cannot track the fast density profile transients due to pellet ablation.

pellet causes a change in the phase of the signal that is not detected by the electronics, yielding no signal increase despite increased line-integrated density. Effectively, this also corresponds to a fringe jump. Consequently the observer can not distinguish between two cases:

- a pellet does not reach the plasma and yields no signal increase on the interferometer,

- a pellet reaches the plasma, and yields no signal increase due to a simultaneous fringe jump that cancels out the signal increase.

On the other hand, the Bremsstrahlung diagnostic can be used for density estimation during pellet injection. The fault handling algorithm is programmed to ignore the interferometers once pellets are launched, and only the Bremsstrahlung signals are used for density estimation. Since no profile shape information can be deduced from Bremsstrahlung measurements alone, the observer relies solely on the RAPDENS model in estimating the profile shape.

In Figure 3.7, the real-time estimated density profiles at four time points and the time traces of the estimated density at four loci of the normalized toroidal 
flux $\rho_{\text {tor }}$ are shown, together with Thomson scattering data points (not available in real time at the time of writing). From the maximum density location of the Thomson scattering data in the top right panel, taken at a pellet arrival, it can be seen that the pellets ablate around $\rho=0.3$.

From the profile plots at $t=3.6 \mathrm{~s}$ and $t=5.2 \mathrm{~s}$, which is during the pellet phase, as well as the time trace of the estimated density at $\rho=0.4$, it can be seen that the observer does not track the local density transients from pellet ablation. The observer's density estimates are only valid in a low-frequency range, since it can not track the fast transients from pellet ablation. This can be seen from the estimated density trace at $\rho=0.4$. The high-frequency signal content originates from the Bremsstrahlung (see Figure 3.6, panel 4 and 5). Also, the profile shape estimates in the pellet injection phase rely only on the internal model and therefore does not feature the local density increase at the ablation location (see e.g. the profile estimate at $t=5.2 \mathrm{~s}$ ). Moreover, towards the end of the pellet phase (around $t=5 \mathrm{~s}$, see the bottom right panel), the observer underestimates the central density, as can be seen in the bottom right panel.

From the bottom left panel, it can be seen that the observer systematically underestimates the edge density. This can also be seen in the third panel of Figure 3.6, where the estimated line-integrated density for the edge line-of-sight of channel H5 is lower than measured. In Section 3.5, the performance and proposed improvements are discussed.

\section{Density control in integrated control exper- iments on TCV}

In this section, the design, implementation and performance of the observer and controller on TCV experiments is presented.

\subsection{Plasma scenario and control problem}

In the considered scenario, the plasma density is required to remain constant despite the presence of disturbances. The physics goal for the profile control experiment described in [Maljaars et al., 2017] is to achieve various targets for the plasma pressure and inverse safety factor profile using EC heating and current

drive. The scenario is a limited L-mode at a low density of about $2 \times 10^{19 \mathrm{~m}^{\frac{3}{3}}}$ to maximize the EC current drive efficiency. The two available gyrotron clusters are aimed at the magnetic axis.

The density controller aims to make the average electron density track a reference. This reference density consists of a ramp followed by a step toward a constant. This constant is the desired density during for the profile control experiment. Reference tracking is to be achieved by a feedback controller using gas injection as the actuator. The controller should: 
- minimize the tracking error $e=x_{\text {ref }}-x_{\mathrm{c}}$ up to a bandwidth of $20 \mathrm{~Hz}$,

- reject disturbances from MHD activity and EC heating and current drive,

- be robust against model mismatches in the model-based tuning procedure.

The controlled quantity is provided in real time by the observer. This observer should:

- minimize the profile estimation error,

- detect and correct for measurement faults,

- be robust against model mismatches.

Moreover, the observer and controller should stay within the available computational time per $1 \mathrm{~ms}$ cycle time of the real-time computer (see Section 4.3). The controller has command over one gas valve. The valve can handle an input voltage between $0 \mathrm{~V}$ and $10 \mathrm{~V}$, yielding a (nonnegative) gas flow into the vacuum vessel. The mapping between input voltage $u_{\text {valve }}$ and mass flow $\Gamma_{\text {valve }}$ has been determined in calibration experiments. This nonlinear function is strictly increasing and can be written as $\Gamma_{\text {valve }}=v\left(u_{\text {valve }}\right)$.

\subsection{Feedback controller design}

The feedback controller is designed using the model-based robust $H_{\infty}$ design formalism [Morari and Zafiriou, 1989]. This approach enables to design the control law based on a linear plant model and requirements for the closed-loop response, and therefore requires minimal experimental time dedicated to controller tuning. Moreover, the robust $H_{\infty}$ control method allows to account for uncertainties in the plant dynamics. Regarding the density dynamics, these uncertainties are formed by shot-to-shot differences in machine conditions, unmodeled (nonlinear) effects and coupling to plasma physics other than solely the density dynamics.

\section{Robust control design procedure}

The main ingredients and outcome of the design procedure are stated next, while the details are provided in Appendix C.

The nonlinear continuous-time dynamics (3.18) are linearized around a nominal operating point $\left(x_{0}, p_{0}\right)$. Uncertainties in the steady-state response and time scales of the dynamics are captured by including static variability in certain parameters and coefficients of the model (3.18). These are listed in Table 3.1.

A set of weighting functions that express the required closed-loop responses are specified by the user. These weighting functions impose zero steady-state tracking and disturbance rejection by penalizing the response of the closed-loop transfer functions in certain frequency ranges. The robust $H_{\infty}$ design procedure for the linear feedback controller subject to the uncertain linearized plant model and the weighting functions is given in Appendix C.3. It is a proportional-integral (PI) controller with additional high-frequency roll-off. The frequency response of the controller is shown in Figure 3.13. 


\section{Anti-windup compensation}

Since the actuation input (the gas valve mass flow) is constrained by a lower limit and an upper limit, an anti-windup scheme has to be employed. This scheme prevents oscillations of the actuation signal (and consequently the plasma density) when the controller has saturated the actuation signal at one of its limits.

The controller (3.39) is implemented as

$$
u_{k}=a u_{k-1}+(1-a)\left(K_{\mathrm{P}} e_{k}+u_{\mathrm{I}, k}\right)
$$

where $K_{\mathrm{P}}$ is the proportional gain and $a=\exp \left(-f_{\text {rolloff }} T_{\mathrm{s}}\right)$ where $f_{\text {rolloff }}$ is the roll-off frequency and $T_{\mathrm{s}}$ is the cycle/sample time. The anti-windup is implemented in the formulation of the integral action $u_{\mathrm{I}, k}$, which is evaluated as

$$
u_{\mathrm{I}, k}= \begin{cases}u_{\mathrm{I}, k-1}+K_{\mathrm{I}} T_{\mathrm{s}} e_{k} & u_{\min }<u_{k-1}<u_{\max } \\ u_{\mathrm{I}, k-1} & u_{\min }>u_{k-1} \vee u_{k-1}>u_{\max }\end{cases}
$$

where $K_{\mathrm{I}}$ is the integral gain. Here, the integration of the error $e$ is halted when the actuator input $u$ is saturated.

\subsection{Implementation on the TCV control system}

The controller and the observer (described in Section 3.2) are implemented on the SCD [Anand et al., 2017], the digital control system of TCV.The observer and controller are designed in the dedicated software environment for development, testing and experimental use of control algorithms [Maljaars et al., 2017].

All real-time software is programmed in MATLAB Simulink [The Mathworks Inc., a], which allows for automated code generation for real-time use [Felici et al., $2014 \mathrm{~b}]$. It is worth to note that the code underlying the observer equations is the same on ASDEX Upgrade and TCV, whereas only the machine-specific diagnostic models and settings differ between the machines. This greatly enhances portability to other implementations.

An overview of the observer and controller implementation on the SCD is given in Figure 3.8. The observer is connected to other real-time algorithms. First, the real-time equilibrium reconstruction code LIUQE [Moret et al., 2015] provides equilibrium geometry information to the RAPTOR-observer and the profile controller, as well as the interferometry matrix to the density observer. Second, the real-time RAPTOR-observer [Felici et al., 2011, 2014a] provides the estimated edge electron temperature. This is estimated by merging soft X-ray measurements of the central electron temperature with the RAPTOR model in an Extended Kalman filter scheme. Also, it estimates the plasma pressure and safety factor profile for the profile controller [Maljaars et al., 2017]. Last, the measured plasma current is provided [Moret et al., 2015]. The real-time algorithms on the SCD mentioned above are run at a cycle time of $1 \mathrm{~ms}$. 


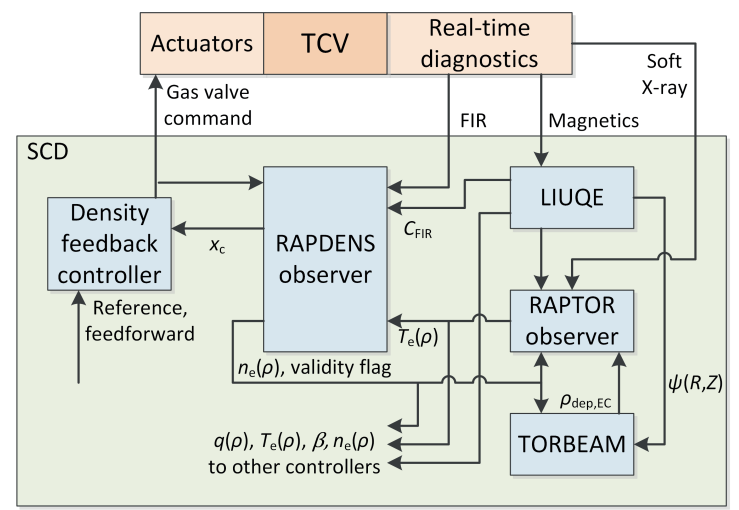

Figure 3.8 - Block diagram of the density observer and controller as implementated on the SCD. Also shown are the RAPTOR-observer and the real-time equilibrium reconstruction code LIUQE. The latter provides the interferometry matrix to the observer. Note that the profile controller for control of the plasma pressure and safety factor profile is not displayed for simplicity.

\section{Forward diagnostics model}

The TCV interferometry system measures the line-integrated electron density along fourteen vertical lines of sight [Barry et al., 1998]. These lines of sight range from (nearly) intersecting the magnetic axis to intersecting the plasma edge, both on the high and low field side. Similar to (3.4), the forward diagnostics model can be written in the form of (3.3) as

$$
y=C_{\mathrm{FIR}} x+\delta d
$$

where $C_{\mathrm{FIR}}^{(i, j)}=\int_{L_{i}} \Lambda_{j} d L$ where $L_{i}$ is the line of sight of channel $i, \delta$ is the lineintegrated density corresponding to one fringe, and $d$ is the cumulative number of fringe jumps. The matrix $C_{\mathrm{FIR}}$ is computed by a postprocessing routine in the implementation of LIUQE [Moret et al., 2015] on the SCD.

\section{Fault handling}

The signals originating from the RAPTOR observer and LIUQE (see Figure 3.8) are checked for validity. For example, if LIUQE has convergence issues (reported by a dedicated output signal) the density observer holds the interferometry matrix $C_{\mathrm{FIR}}$, plasma volume $V_{\mathrm{p}}$, and $\mathrm{X}$-point presence indicator $c_{\mathrm{LD}}$ to their previous valid values.

In order to filter out fringe jumps, the innovation residual $z_{k}$ of the observer (see Appendix B) is fed through a filter that removes multiples of one fringe. This fringe jump correction scheme is documented in [Blanken et al., 2018b]. 


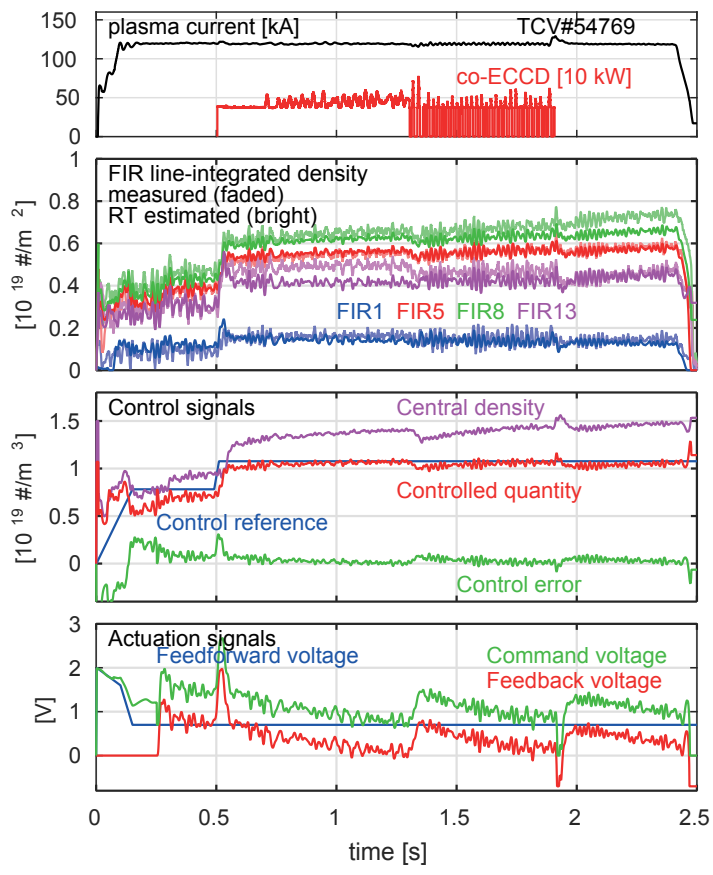

Figure 3.9 - Real-time results for TCV discharge \#54769. First panel: plasma current. Second panel: measured interferometer signals and estimated lineintegrals (computed from the estimated density profile). Third panel: controlled variable, reference, central density and control error. Fourth panel: feedforward, feedback and command (feedforward plus feedback) signals for the gas valve. In this experiment, the density averaged over $\rho_{\mathrm{v}}$ is controlled by a combination of feedforward and feedback. At $t=0.25 \mathrm{~s}$, the feedback controller introduced in Section 4.2 is activated. The estimated density profiles and Thomson scattering data are shown in Figure 3.10.

\subsection{Experimental results of density control}

In this subsection, the results of the observer and controller are presented. In these experiments the density is required to stay constant while EC heating and current drive is used to control the plasma pressure and safety factor profile (see [Maljaars et al., 2017]). The controlled variable $x_{\mathrm{c}}$ is selected to be the electron density averaged over $\rho_{\mathrm{v}}=\sqrt{\frac{V}{V_{\mathrm{p}}}}$, i.e. $\bar{n}_{\mathrm{e}}=\int_{0}^{1} n_{\mathrm{e}} d \rho_{\mathrm{v}}=$ $\frac{1}{2 \sqrt{V_{\mathrm{p}}}} \int_{0}^{1} \frac{n_{\mathrm{e}}}{V} \frac{\partial V}{\partial \rho_{\mathrm{tor}}} d \rho_{\text {tor }}$. This quantity is somewhat equivalent to a line-average (as commonly derived from interferometry measurements), yet is not dependent on the machine-specific interferometer geometry.

In TCV discharge \#54769, the feedback controller is activated at $t=0.25 \mathrm{~s}$. 

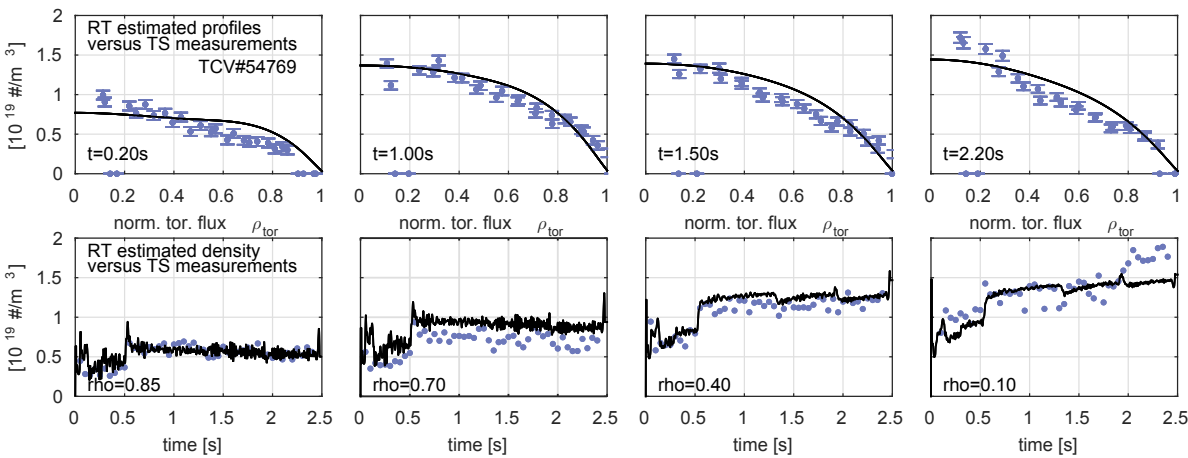

Figure 3.10 - Real-time estimated density profiles with offline Thomson Scattering measurements for TCV shot \#54769. Top row: estimated profiles at four points in time. Bottom row: time traces at four loci of the normalized toroidal flux $\rho_{\text {tor }}$. The agreement between the estimated density and Thomson scattering data is good, despite an error in the overall profile shape. This can be attributed to the accuracy of the chosen transport and source models (see Section ??) as well as unknown errors in the evaluation of line-integrals from real-time reconstructed equilibria.

The legacy controller [Vijvers et al., 2012] is active between 0.1s and 0.25s. A model-predictive controller [Maljaars et al., 2017] is used to control the safety factor profile using one EC cluster driving co-current. In Figure 3.9, the plasma current, EC powers and interferometry signals are shown, together with the realtime estimations from the observer. Also, the control reference and gas valve commands are shown. After initial transients, a zero tracking error $e$ is achieved. In Figure 3.10, the real-time estimated density profiles and the time traces of the estimated density at four loci of the normalized toroidal flux $\rho_{\text {tor }}$ are shown, together with Thomson scattering data points. These comparisons show that the observer provides an estimate with good accuracy.

In TCV discharge \#55117, the feedback controller is activated at $t=0.2 \mathrm{~s}$. A controller originally developed for ITER is used (see [Kim et al., 2016]) to control the plasma pressure using one EC cluster driving co-current. In Figure 3.11, the plasma current, EC powers, rotating mode markers and interferometry signals are shown, together with the real-time estimations from the observer. Also, the control reference and gas valve commands are shown. In Figure 3.12, the realtime estimated density profiles and the time traces of the estimated density at four loci of the normalized toroidal flux $\rho_{\text {tor }}$ are shown, together with Thomson scattering data points.

After initial density transients, the tracking error $e$ decays to zero in the flat-top phase. At $t=1.77 \mathrm{~s}$, a rotating mode forms. The resulting thermal confinement deterioration prompts the pressure controller to increase the EC 

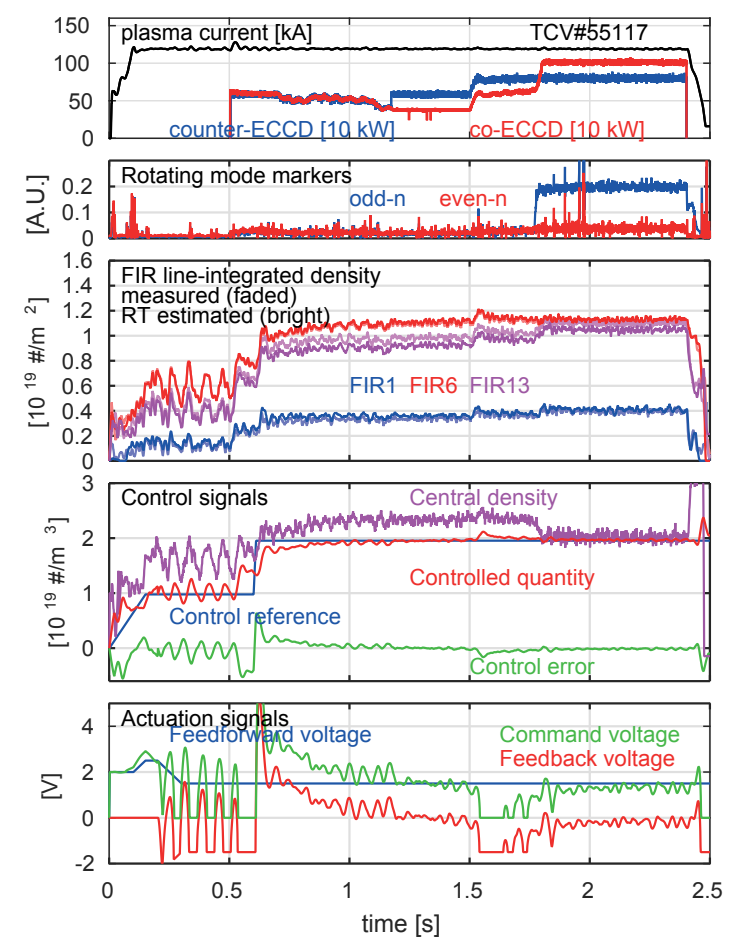

Figure 3.11 - Real-time results for TCV discharge \#55117. First panel: plasma current. Second panel: presence markers of rotating modes. Third panel: measured interferometer signals and estimated line-integrals (computed from the estimated density profile). Fourth panel: controlled variable, reference, central density and control error. Fifth panel: feedforward, feedback and command signals for the gas valve. Again, the density averaged over $\rho_{\mathrm{v}}$ is controlled by a combination of feedforward and feedback. From the start of the discharge until $t=0.25 \mathrm{~s}$, the legacy density controller is active. At $t=0.25 \mathrm{~s}$, the feedback controller introduced in Section 4.2 is activated. The control error decreases to zero for the duration of the discharge. The estimated density profiles and Thomson scattering data are shown in Figure 3.12.

heating. At the same time, a change in the density profile shape is observed on the interferometers, as the HFS edge interferometer (\#13) increases while a central and the LFS edge interferometer (\#6 and \#1, respectively) do not. The observer interprets this as a slightly hollow profile (see the profile at $t=2.0 \mathrm{~s}$ in Figure 3.12). Interestingly, the Thomson scattering data does not show a similar hollow profile.

Between $t=0.2 \mathrm{~s}$ and $t=0.5 \mathrm{~s}$, oscillations are seen in the interferometry measurements, estimated density as well as the feedback actuation. After the 

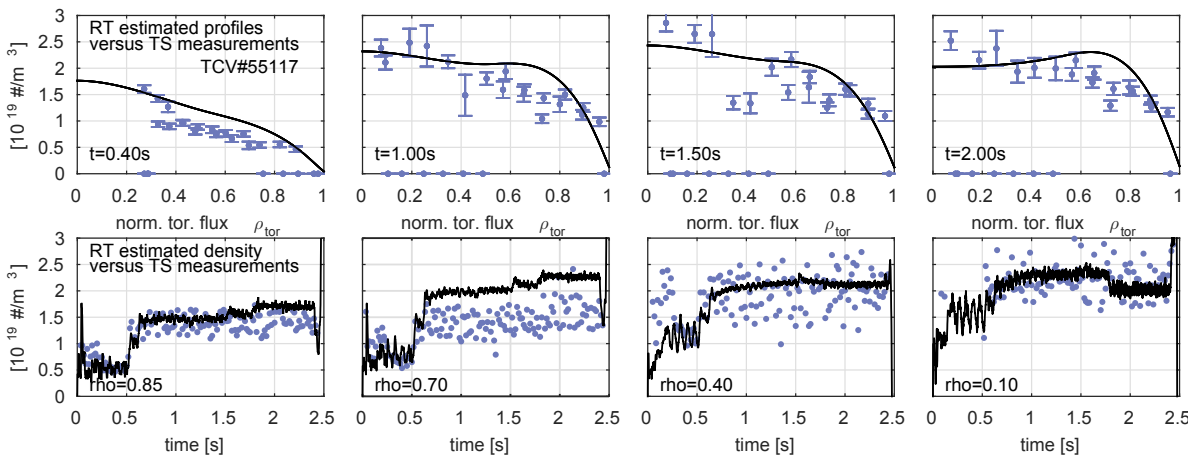

Figure 3.12 - Real-time estimated density profiles with offline Thomson Scattering measurements for TCV shot \#55117. Top row: estimated profiles at four points in time. Bottom row: time traces at four loci of the normalized toroidal flux $\rho_{\text {tor }}$.

EC heating switches on, these oscillations are smaller and decay. Note that the oscillation are not caused by integrator windup, as it takes one period of the oscillation before the actuation command signal saturates at $0 \mathrm{~V}$ for the first time. Multiple reasons could cause such oscillations.

- The feedback controller gains could be too aggressive for the plasma response in the ohmic phase. The gains prove satisfactory in the EC-heated phase, where the oscillation is much smaller. Note that the controller is tuned on a linearized model (see Appendix C.3), linearized at an operating point in the EC-heated phase.

- The plasma dynamics of the pressure and density are coupled. The interconnection of the state observers and controllers for the pressure and density, as well as their respective computational delays, could cause the two feedback loops to become oscillatory.

Additional results of discharges with the same scenario, pressure and safety factor profile control, and density control can be found in [Maljaars et al., 2017; Mavkov et al., 2018].

\section{Discussion}

In this section, we provide a discussion of the estimation and control performance in experiments that are shown in Section 3.3 and Section 3.4. 


\subsection{Profile estimation accuracy}

In Section 3.3 and 3.4, we have shown good agreement between the real-time estimated density profiles and offline Thomson Scattering measurements.

In the high-density pellet-fuelled experiments on ASDEX Upgrade, the observer systematically underestimates the edge density and does not track fast local density transients due to pellet ablation. Since many interferometers give invalid signals in this experiment, the estimate is based on a limited set of Bremsstrahlung measurements. The underestimation did not prove harmful for the experimental goal.

Moreover, the systematic integration of multiple diagnostics in a real-time estimator is novel in present-day tokamaks and will be essential in ITER and DEMO due to scarce diagnostics and harsh environment. Future addition of real-time Thomson scattering measurements can alleviate the discrepancy at the edge.

\subsection{Control performance}

The real-time estimated central density proved essential for the feedback control in the high density scenarios on ASDEX Upgrade (see [Lang et al., 2018]). The central density was maintained above the Greenwald limit for more than 2s. While alternative real-time reconstructions of the central density proved unreliable in earlier discharges (see [Lang et al., 2012, 2018]), the estimation accuracy of the proposed method is better and sufficient for the purpose of control in this experiment.

In experiments on the simultaneous control of density, pressure and safety factor profile on TCV, the feedback controller was tuned a priori using the modeled plasma response of the actuator to the controlled variable. Such a model-based tuning practice is advised for ITER and DEMO, where experimental time can not be used for controller tuning by trial and error.

Although strict requirements for control performance are not yet formed, the controller algorithms proved successful for the experimental goals.

\subsection{Future extensions}

The accuracy and robustness of real-time estimated profiles, both in the plasma core and in the edge, can be enhanced by incorporating additional diagnostics, as well as improved transport modeling. While modeling accuracy of particle transport internal to the plasma has progressed in recent years [Angioni et al., 2009], the real-time capable self-consistent modeling of recycling and pumping is still not well understood and currently requires large-scale simulations using numerical codes such as SOLPS [Wiesen et al., 2015].

The inclusion of real-time available Thomson scattering measurements on ASDEX Upgrade and TCV will greatly improve the profile estimation accuracy. 
In particular, a Thomson scattering measurement sample fired after a pellet launch can prove useful for correcting any fringe jumps on the interferometers. Also, the inclusion of real-time dedicated plasma edge diagnostics will improve accuracy and robustness of the estimation of the pedestal density gradient, which is important for e.g. bootstrap current estimation.

\section{Conclusions}

Reliable real-time plasma density profile estimation is essential for the operation of next-generation tokamaks and nuclear fusion reactors. An integrated solution using all available yet scarce diagnostics should be in place to provide an accurate estimate of the electron density profile in real time. This is crucial for density control, and valuable for determining diagnostics validity and actuation constraints (e.g. ECE, ECH cut-off), and control loops for pressure and MHD.

In this paper, the successful results of a real-time density profile estimator and diagnostic fault handling algorithm in experiments on ASDEX Upgrade and TCV are presented. This observer uses multiple diagnostics and can deal with various diagnostic faults. In ASDEX Upgrade discharges on high-density pellet fuelling, the observer provides good agreement with offline Thomson scattering measurements, and provides a reliable estimate of the controlled core density for the feedback controller. In TCV discharges on integrated pressure, iota and NTM control, the density controller provides a constant density despite disturbances from time-varying ECCD power. Here also, the real-time estimated density profile shows sufficiently good agreement with Thomson scattering measurements. Shortcomings in estimation quality can be attributed to limitations of the model as well as unmodeled diagnostics characteristics.

The observer has well-defined validity limits. The estimation accuracy deteriorates when these limits are exceeded. False positives of fringe jumps occur when the signal resembles one. Also, pellet-induced transients and fringe jumps are inherently difficult to distinguish. Future work on pellet modeling and real-time pellet arrival detection can solve these difficulties.

On the one hand, given this experience, it will be important for ITER that the real-time estimated density is based on all available diagnostics to achieve accuracy, reliability and redundancy. On the other hand, future modeling advances and knowledge gained from ITER will allow real-time density estimation from a more limited set of diagnostics, such as is the case on DEMO.

\section{Acknowledgments}

This work has been carried out within the framework of the EUROfusion Consortium and has received funding from the EURATOM research and training 
programme 2014-2018 under grant agreement No 633053. The views and opinions expressed herein do not necessarily reflect those of the European Commission. This work was supported in part by the Netherlands Organization for Scientific Research via F. Felici's VENI grant "Control of plasma profiles in a fusion reactor" (no. 680.47.436). This work was supported in part by the Swiss National Science Foundation.

\section{Appendix}

\section{A Equation summary of RAPDENS model}

In this appendix, a summary of the RAPDENS equations, governing the evolution of the plasma electron density, and its spatial and temporal discretization is given.

The evolution of the plasma density $n_{e}(\rho, t)$ is modeled by mass conservation and radial transport [Hinton and Hazeltine, 1976] on the domain $0<\rho<\rho_{\mathrm{e}}$ as

$$
\left(\frac{\partial V}{\partial \rho}\right)^{-1} \frac{\partial}{\partial t}\left(n_{\mathrm{e}} \frac{\partial V}{\partial \rho}\right)+\left(\frac{\partial V}{\partial \rho}\right)^{-1} \frac{\partial \Gamma_{\mathrm{e}}}{\partial \rho}=S_{\mathrm{e}}
$$

where $\rho=\sqrt{\Phi /\left.\Phi\right|_{\text {LCFS }}}$ is the radial variable associated with the toroidal flux, $\rho_{\mathrm{e}}>1$ is an artificially extended boundary to include a scrape-off layer volume (see [Blanken et al., 2018b]) and $\frac{\partial V}{\partial \rho}$ is the radial volume derivative. The radial transport flux $\Gamma_{\mathrm{e}}(\rho, t)$ is modeled as

$$
\Gamma_{\mathrm{e}}=-\frac{\partial V}{\partial \rho}\left(\left\langle(\nabla \rho)^{2}\right\rangle \chi \frac{\partial n_{e}}{\partial \rho}+\langle|\nabla \rho|\rangle \nu n_{e}\right)
$$

where $\chi$ is the diffusivity and $\nu$ is the drift velocity. The boundary conditions for (3.10) are $\left.\frac{\partial n_{e}}{\partial \rho}\right|_{\rho=0}=0$ and $\left.n_{e}\right|_{\rho=\rho_{\mathrm{e}}}=0$. The net electron source $S_{\mathrm{e}}(\rho, t)$ is modeled as

$$
S_{\mathrm{e}}=\langle\sigma v\rangle_{\mathrm{iz}} n_{n} n_{e}-\langle\sigma v\rangle_{\mathrm{rec}} n_{e} n_{i}-\frac{\left.n_{e}\right|_{\rho>1}}{\tau_{\mathrm{SOL}}}+S_{\mathrm{pellet}}+S_{\mathrm{NB}}
$$

where $\langle\sigma v\rangle_{\text {iz }}\left(T_{\mathrm{e}}\right)$ and $\langle\sigma v\rangle_{\text {rec }}\left(T_{\mathrm{e}}\right)$ are the temperature-dependent ionization and recombination cross-sections [Wesson, 2004], and $\tau_{\mathrm{SOL}}$ is the time constant for particle loss through the scrape-off layer. These sources and sinks arise from particle flows between the plasma, the vacuum and the wall. The evolution of the neutral vacuum density $n_{\mathrm{n}}(t)$ and wall inventory $N_{\mathrm{w}}(t)$ are modeled as

$$
\frac{d N_{\mathrm{w}}}{d t}=-\frac{N_{\mathrm{w}}-c_{w} V_{\mathrm{v}, 0} n_{\mathrm{n}}}{\tau_{\text {release }}}+\left(1-\frac{N_{\mathrm{w}}}{N_{\mathrm{sat}}}\right) \int_{V_{\mathrm{SOL}}} \frac{n_{e}}{\tau_{\mathrm{SOL}}} d V
$$




$$
\begin{aligned}
V_{\mathrm{v}} \frac{d n_{\mathrm{n}}}{d t}=\quad & \frac{N_{\mathrm{w}}-c_{w} V_{\mathrm{v}, 0} n_{\mathrm{n}}}{\tau_{\text {release }}}+\frac{N_{\mathrm{w}}}{N_{\mathrm{sat}}} \int_{V_{\mathrm{SOL}}} \frac{n_{e}}{\tau_{\mathrm{SOL}}} d V \\
+\int_{V_{\mathrm{p}}} & \left(\langle\sigma v\rangle_{\mathrm{rec}} n_{e} n_{i}-\langle\sigma v\rangle_{\mathrm{iz}} n_{n} n_{e}\right) d V \\
& -\frac{n_{\mathrm{n}} V_{\mathrm{v}, 0}}{\tau_{\mathrm{pump}}}+\left.\Gamma\right|_{\rho_{\mathrm{e}}}+\Gamma_{\text {valve }}
\end{aligned}
$$

where $\tau_{\text {release }}$ is the particle release rate from the wall, $N_{\text {sat }}$ is the inventory at which the wall is saturated, $\tau_{\text {pump }}$ is the pumping time scale, $c_{w} V_{v, 0}, V_{\mathrm{v}}$ is the vacuum volume and $V_{\mathrm{SOL}}=\int_{1}^{\rho_{\mathrm{e}}} \frac{\partial V}{\partial \rho} d \rho$.

The equilibrium-related quantities are parametrized as

$$
\begin{array}{r}
\frac{\partial V}{\partial \rho}=\left.2 V\right|_{\text {LCFS }}(t) \rho \\
\left\langle(\nabla \rho)^{2}\right\rangle=\left(g_{1}-1\right) \rho^{g_{3}}+1 \\
\langle|\nabla \rho|\rangle=\left(g_{2}-1\right) \rho^{g_{4}}+1
\end{array}
$$

where $g_{1}=\left.\left\langle(\nabla \rho)^{2}\right\rangle\right|_{\text {LCFS }}, g_{2}=\left.\langle|\nabla \rho|\rangle\right|_{\text {LCFS }}, g_{3}$ and $g_{4}$ are derived from a fixed equilibrium. Also, distributed time-varying quantities are parametrized as e.g. $S_{\mathrm{NB}}=\Lambda_{\mathrm{NB}}(\rho) \Gamma_{\mathrm{NB}}(t)$, and similar for the pellet deposition $S_{\text {pellet }}$ and the neutral density $n_{\mathrm{n}}$ in the plasma, see also [Blanken et al., 2018b].

A system of ordinary differential equations (ODE) is constructed in [Blanken et al., 2018b] by parametrizing the spatial dependency of the electron density $n_{e}(\rho, t)$ (as expressed in equation (3.1)) using cubic splines [de Boor, 2001] and applying a finite-element method (see e.g. [Hughes, 1987]) to (3.10), (3.13) and (3.14). This system of ODEs is written as

$$
\frac{d x}{d t}=g(x, p, u)
$$

where $x(t)$ is the state vector containing $N_{\mathrm{w}}(t), n_{\mathrm{n}}(t)$ and the parametrization coefficients for $n_{e}(\rho, t), u(t)=\left[\Gamma_{\text {valve }}(t) \Gamma_{\mathrm{NB}}(t) \Gamma_{\text {pellet }}(t)\right]^{\mathrm{T}}$ is the input vector and $p(t)$ is the parameter vector, containing time-varying quantities $I_{\mathrm{p}}, T_{e}$, $V_{\mathrm{LCFS}}, c_{\mathrm{LD}}, c_{\mathrm{LH}}$, see [Blanken et al., 2018b]. A backward Euler scheme with constant time step $T_{\mathrm{S}}$ is employed to transform (3.18) to the nonlinear ${ }^{3}$ discretetime equation

$$
f\left(x_{k}, x_{k-1}, p_{k-1}, u_{k-1}\right)=-T_{\mathrm{s}} g\left(x_{k}, p_{k-1}, u_{k-1}\right)+x_{k}-x_{k-1}=0
$$

where $x_{k}=x\left(t_{k}\right)$ denotes the time-discretized vector and $t_{k}=t_{0}+k T_{\mathrm{s}}, k=$ $0,1, \cdots, t_{\mathrm{f}} / T_{\mathrm{s}}$. Linearizations of both $g$ in (3.18) and $f$ in (3.19) can be analytically derived. In the applications described in this paper, equation (3.19) is solved using a Newton-Raphson method.

\footnotetext{
${ }^{3}$ Note that for the modeling presented in this paper, the function $g$ in (3.18) is in fact linear with respect to $u$.
} 


\section{B Equation summary of the observer equa- tions}

In this appendix, the observer equations based on an Extended Kalman filter for the RAPDENS dynamical model (3.19) and measurement equation (3.3) are stated.

\section{B.1 Augmented system equations}

The state of the system (which is to be estimated by the observer) is composed of three vectors. In the Kalman filter formalism, the state evolution and measurement are assumed to be corrupted by stochastic noise. The three parts of the state and the respective stochastic noise terms are listed below.

1. The first part is the state $x_{k}$ of the RAPDENS dynamics (3.19). A term $w_{k}^{\mathrm{x}}$ is added in order to handle model uncertainties that influence the state evolution. This term is assumed to be a zero-mean white noise with covariance $Q_{k}^{\mathrm{x}}$.

2. The second part is the vector of coefficients $\zeta_{k}$ in (3.3). In Section $3.2, \zeta_{k}$ is assumed to be slowly changing with respect to the state of the system. This allows them to be estimated by the observer. It is assumed to have random walk dynamics as expressed by $\zeta_{k}=\zeta_{k-1}+w_{k}^{\mathrm{z}}$, where $w_{k}^{\mathrm{z}}$ is assumed to be zero-mean white noise with covariance $Q_{k}^{z}$.

3. The third part is the number of fringe jumps on each channel of the interferometry system (see Section 3.2 and Section 4.3). It must be stored over time in order to compensate the measurement $y_{\text {FIR }}$ at every time step following such a jump. In Section 3.2, the influence of fringe jumps on the interferometry measurements is written as an additive term $\delta d_{k}$, where $\delta$ is a constant. This state vector $d_{k}$ accumulates the jumps on each chan-nel as $d_{k}$ $=d_{k-1}+\Delta_{k-1}$, where $\Delta_{k} \in \mathbb{N}$. For example, a downward fringe jump on channel $i$ at time $t_{k}$ is represented by $\Delta_{k}^{(i)}=-1$.

Summarizing, the equations governing the state transitions are

$$
\begin{array}{r}
f\left(x_{k}, x_{k-1}, p_{k-1}, u_{k-1}\right)-\frac{\partial f}{\partial x_{k}} w_{k-1}^{\mathrm{x}}=0 \\
\zeta_{k}=\zeta_{k-1}+w_{k-1}^{\mathrm{z}} \\
d_{k}=d_{k-1}+\Delta_{k}
\end{array}
$$

Note that in $(3.20)$, the additive term $w_{k}^{\mathrm{x}}$ is multiplied by the derivative of $f$ to $x_{k}$. If $f$ would be linear with respect to its arguments, as is the case for linear dynamical systems, then the usual state-space form with additive term as used in e.g. [Anderson and Moore, 1979] is recovered. This implies that (3.20) 
represents a nonlinear state transition with additive noise, as is assumed in the Extended Kalman filter formalism [Anderson and Moore, 1979].

The measurement is assumed to be corrupted by a zero-mean noise $v_{k}$ with covariance $R_{k}$, and is given by

$$
y_{k}=h\left(x_{k}, \zeta_{k}\right)+\delta d_{k}+v_{k}
$$

where the additive terms $w_{k}^{\mathrm{x}}, w_{k}^{\mathrm{z}}$ and $v_{k}$ are assumed to be zero-mean white noise with covariances $Q_{k}^{\mathrm{x}}, Q_{k}^{\mathrm{z}}$ and $R_{k}$, respectively.

The Extended Kalman filter relies on local linearizations of the relevant dynamics and measurement relation. For the purpose of readability later on, three functions related to local linearizations of (3.20) and (3.23) are defined as $\tilde{f}\left(x_{k}, x_{k-1}, p_{k-1}\right)=-\left.\left(\left.\frac{\partial f}{\partial x_{k}}\right|_{x_{k}, p_{k-1}}\right)^{-1} \frac{\partial f}{\partial x_{k-1}}\right|_{x_{k-1}, p_{k-1}}, \tilde{h}_{\mathrm{x}}\left(x_{k}, \zeta_{k}\right)=\left.\frac{\partial h}{\partial x}\right|_{x_{k}, \zeta_{k}}$ and $\tilde{h}_{\mathrm{z}}\left(x_{k}, \zeta_{k}\right)=\left.\frac{\partial h}{\partial \zeta}\right|_{x_{k}, \zeta_{k}}$. Moreover, an augmented state is defined by stacking $x_{k}$ and $\zeta_{k}$ as $\boldsymbol{x}_{k}=\left[\begin{array}{cc}x_{k}^{T} & \zeta_{k}^{T}\end{array}\right]^{T}$.

\section{B.2 Observer equations based on an Extended Kalman filter}

The observer equations consist of two distinctive parts: the model-based state prediction and the measurement-based state update. The state prediction step is based on (3.20)-(3.22) and is given by

$$
\begin{array}{r}
f\left(\hat{x}_{k \mid k-1}, \hat{x}_{k-1 \mid k-1}, p_{k-1}, u_{k-1}\right)=0 \\
\hat{\zeta}_{k \mid k-1}=\hat{\zeta}_{k-1 \mid k-1} \\
\hat{d}_{k \mid k-1}=\hat{d}_{k-1 \mid k-1}
\end{array}
$$

The covariance matrix $P_{k \mid k-1}$ of the prediction error of the augmented state $\hat{\boldsymbol{x}}_{k \mid k-1}$ is given by

$$
P_{k \mid k-1}=F_{k-1} P_{k-1 \mid k-1} F_{k-1}^{T}+Q_{k-1}
$$

where the matrix $F_{k}$ is derived from a local linearization of (3.20) and is given by

$$
F_{k-1}=\left[\begin{array}{cc}
\tilde{f}\left(\hat{x}_{k \mid k-1}, \hat{x}_{k-1 \mid k-1}, p_{k-1}\right) & 0 \\
0 & I
\end{array}\right]
$$

where $I$ is the identity matrix and $Q_{k}$ is a block-diagonal matrix with blocks $Q_{k}^{\mathrm{x}}$ and $Q_{k}^{\mathrm{z}}$.

In the update step, the predicted estimate is adjusted according the measurement sample $y_{k}$. The innovation residual $z_{k}$ is the difference between the measurement sample and the prediction of the measured quantity. The latter is 
computed on the output equation (3.23). The innovation residual, its covariance matrix $S_{k}$ and the near-optimal Kalman gain $L_{k}$ are given by

$$
\begin{aligned}
z_{k} & =y_{k}-h\left(\hat{x}_{k \mid k-1}, \hat{\zeta}_{k \mid k-1}\right)-\delta \hat{d}_{k \mid k-1} \\
S_{k} & =R_{k}+H_{k} P_{k \mid k-1} H_{k}^{T} \\
L_{k} & =P_{k \mid k-1} H_{k}^{T} \mathbb{S}_{k}^{T}\left(\mathbb{S}_{k} S_{k} \mathbb{S}_{k}^{T}\right)^{-1}
\end{aligned}
$$

where the matrix $H_{k}$ is derived from local linearizations of (3.23) and is given by $H_{k}=\left[\tilde{h}_{\mathrm{x}}\left(\hat{x}_{k \mid k-1}, \hat{\zeta}_{k \mid k-1}\right) \quad \tilde{h}_{\mathrm{z}}\left(\hat{x}_{k \mid k-1}, \hat{\zeta}_{k \mid k-1}\right)\right]$ and $\mathbb{S}_{k}$ is a selection matrix. Its purpose is discussed later on.

Finally, the updated estimate $\hat{\boldsymbol{x}}_{k \mid k}$ of the augmented state, its covariance matrix $P_{k \mid k}$ and the estimated fringe jump state $\hat{d}_{k \mid k}$ are given by

$$
\begin{aligned}
\hat{\boldsymbol{x}}_{k \mid k} & =\hat{\boldsymbol{x}}_{k \mid k-1}+L_{k} \mathbb{S}_{k}\left(z_{k}-\delta \hat{\Delta}_{k}\right) \\
\hat{d}_{k \mid k} & =\hat{d}_{k \mid k-1}+\hat{\Delta}_{k} \\
P_{k \mid k} & =\left(I-L_{k} \mathbb{S}_{k} H_{k}\right) P_{k \mid k-1}
\end{aligned}
$$

where $\hat{\Delta}_{k}$ is the vector of the estimated number of fringes jumped at time $k$ at each interferometer channel. The expressions for $\hat{\Delta}_{k}$ on the ASDEX Upgrade implementation is given in equation (3.6), whereas for the TCV case it is given in [Blanken et al., 2018b].

In the diagnostic fault handling scheme of Section 3.2, at each time step $k$ the validity of a measurement is assessed. If invalid, the measurement must be ignored by the observer in the update step. This is accomplished in the observer equations by a selection matrix $\mathbb{S}_{k}$ in equations (3.30), (3.31) and (3.33). The matrix $\mathbb{S}_{k}$ is constructed at every time step $k$ by taking an identity matrix with the dimension of the total number of measurement channels and removing the rows corresponding to the invalid channels. The selection matrix $\mathbb{S}_{k}$ ensures that the state estimate is only updated using valid measurements in (3.31) and (3.33). Note that this implies that the Kalman gain matrix $L_{k}$ constructed in (3.30) and $\mathbb{S}_{k}$ have time-varying dimensions.

In the observer implementations described in Section 3.2 and Section 4.3, the recursive equations (3.24)-(3.33) are computed at every cycle step. This is illustrated in Figure 3.1.

In the figures of Section 3.3 and 4.4, estimated diagnostics signals are shown. These are evaluated using (3.23) as

$$
\hat{y}_{k \mid k}=h\left(\hat{x}_{k \mid k}, \hat{\zeta}_{k \mid k}\right)+\delta \hat{d}_{k \mid k}
$$

and represent the expected diagnostic signal based on the updated state estimates of (3.31) and (3.32). 
Table 3.1 - Nominal values and uncertainty interval of uncertain coefficients and parameters in the model (3.18) for the TCV target scenario.

\begin{tabular}{|l|l|l|}
\hline Coefficient / parameter & Unit & Nominal value with uncertainty interval \\
\hline \hline$\tau_{\mathrm{SOL} \rightarrow \text { wall }}$ & $\mathrm{s}$ & $2.3 \times 10^{-4} \pm 30 \%$ \\
\hline$b$ & $\frac{\# e}{\mathrm{~s}} \frac{\mathrm{Ls}}{\mathrm{mbar}}$ & $2.47 \pm 10 \%$ \\
\hline$N_{\mathrm{sat}}$ & $\# \mathrm{e}$ & $2.5 \times 10^{19} \pm 50 \%$ \\
\hline$T_{\mathrm{e}}(\rho=1)$ & $\mathrm{eV}$ & $50 \pm 10 \%$ \\
\hline$V_{\mathrm{p}}$ & $\mathrm{m}^{3}$ & $1.6 \pm 20 \%$ \\
\hline
\end{tabular}

\section{Details of controller design}

In this appendix, the design of a feedback controller using $H_{\infty}$ synthesis tools is elaborated. This state feedback controller stabilizes the closed-loop and achieves robust performance against the modeled uncertainties.

\section{C.1 Uncertain plant model}

The continuous-time model (3.18) is linearized around a nominal stationary operating point $x_{0}, u_{0}$ and written as

$$
\frac{d \tilde{x}}{d t}=A \tilde{x}+B \tilde{u}=\left.\frac{d g}{d x}\right|_{x_{0}, u_{0}} \tilde{x}+\left.\frac{d g}{d u}\right|_{x_{0}, u_{0}} \tilde{u}
$$

where $\tilde{x}=x-x_{0}$ and $\tilde{u}=u-u_{0}$. Next, a subset of the parameters in $p$ and coefficients (see Appendix A) is considered uncertain with nominal values $p, c$ and uncertainty sets $\Delta p, \Delta c$. The system matrix and input matrix of the uncertain plant model are $A_{\text {unc }}=\left.A\right|_{p, c}$ and $B_{\text {unc }}=\left.B\right|_{c}$ where $p$ and $c$ are samples from the uncertainty sets $p+\Delta p$ and $c+\Delta c$. The nominal values and uncertainties considered in this work are shown in Table 3.1. These correspond to uncertainty in the steady-state gain and time scales of (3.35).

The controlled variable is defined as a linear combination of the plant states and is defined as $x_{\mathrm{c}}=Z \tilde{x}$. The transfer function of the uncertain plant model is denoted by $G(s)$ and is given by

$$
G(s)=Z\left(s I-A_{\text {unc }}\right)^{-1} B_{\text {unc }}
$$

The frequency response of $G$ for various values in the uncertainty set is shown in Figure 3.13. Recall that the control error is defined as $e=x_{\text {ref }}-x_{\mathrm{c}}$ where $x_{\text {ref }}$ denotes the reference. We define $P$ to be the open-loop tracking interconnection 


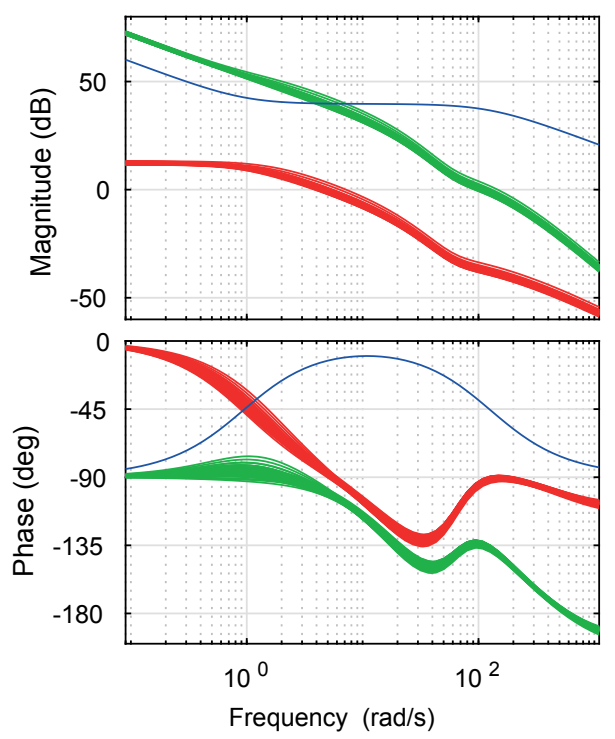

Figure 3.13 - Bode diagram of various frequency responses of the uncertain plant $G(j \omega)$ (red), controller $K(j \omega)$ (blue) and open-loop $L(j \omega)$ (green). Note that the low-frequent response of the plant $G$ has a stable pole around $1 \mathrm{rad} / \mathrm{s}$. The pole location and steady-state response vary due to the uncertain coefficients and parameters.

of the plant $G$. This interconnection is given by

$$
\left[\begin{array}{c}
e \\
\tilde{u} \\
x_{c} \\
e
\end{array}\right]=P\left[\begin{array}{c}
x_{\mathrm{ref}} \\
\tilde{u}
\end{array}\right]=\left[\begin{array}{cc}
I & -G \\
0 & I \\
0 & G \\
I & -G
\end{array}\right]\left[\begin{array}{c}
x_{\mathrm{ref}} \\
\tilde{u}
\end{array}\right]
$$

\section{C.2 Weighting filters}

In order to impose reference tracking below the required bandwidth $\omega_{b}$, zero steady-state error and disturbance rejection, the tracking interconnection $P$ with weighting filters. The augmented tracking interconnection is given by

$$
P_{W}=\left[\begin{array}{llll}
W_{S} & & & \\
& W_{R} & & \\
& & W_{T} & \\
& & & I
\end{array}\right] P
$$




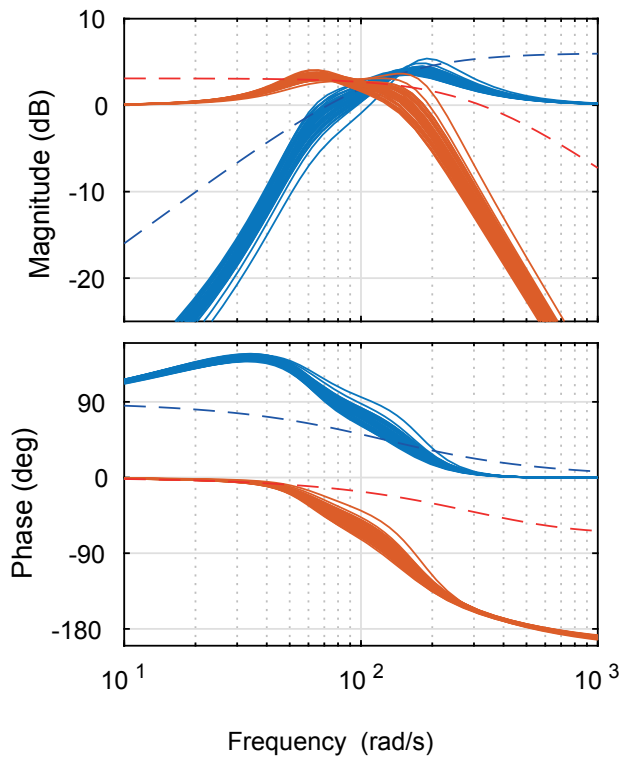

Figure 3.14 - Bode diagram of control sensitivity $S(j \omega)$ (blue) (and inverse shaping function $W_{S}^{-1}(j \omega)$ in dashed blue), complementary control sensitivity $T(j \omega)$ (red) (and inverse shaping function $W_{T}^{-1}(j \omega)$ in dashed red). The sensitivity crosses the $0 \mathrm{~dB}$ between $65 \mathrm{rad} / \mathrm{s}$ and $100 \mathrm{rad} / \mathrm{s}$.

where $W_{S}(s), W_{R}(s)$ and $W_{T}(s)$ are the weighting filters. Here, $W_{S}(s)$ imposes zero steady-state error, penalizes tracking error below the bandwidth $\omega_{b}$ and penalizes the sensitivity above $6 \mathrm{~dB}, W_{T}(s)$ penalizes closed-loop gain above $3 \mathrm{~dB}$ and $W_{R}(s)$ penalizes control action over all frequencies. The inverse of the filters $W_{S}(s)$ and $W_{T}(s)$ are shown in Figure 3.14.

\section{C.3 Controller synthesis by $H_{\infty}$ norm minimization}

A controller of the form

$$
K(s)=\frac{\left(K_{\mathrm{P}} s+K_{\mathrm{I}}\right) \omega_{\text {rolloff }}}{s\left(s+\omega_{\text {rolloff }}\right)}
$$

is chosen. The gains $K_{\mathrm{P}}$ and $K_{\mathrm{I}}$ are to be determined by the synthesis, while $\omega_{\text {rolloff }}$ is fixed at $40 \pi \mathrm{rad} / \mathrm{s}$. The closed-loop interconnection $F$ is defined as $F=$ $P_{W} \star K$ where $\star$ denotes the Redheffer star product. The free gains are then tuned by minimizing the $H_{\infty}$-norm of $F$ using the MATLAB function hinf struct from the Robust Control toolbox [The Mathworks Inc., a]. The resulting controller $K$ stabilizes the closed-loop interconnection and achieves robust performance against the uncertainties. In Figure 3.13, the frequency response of the controller 
$K$ and open-loop transfer $L=G K$ are plotted. In Figure 3.14, the sensitivity $S$ and complementary sensitivity $T$ are plotted. These are defined as $S=\frac{I}{I-L}$ and $T=\frac{L}{I-L}$. These bode plots show that the sensitivity and complementary sensitivity have the desired shape, since the magnitude response is smaller than those of their respective inverse weighting filters. 